\title{
Beam breakup growth and reduction experiments in long-pulse electron beam transport
}

\author{
P. R. Menge, ${ }^{\text {a) }}$ R. M. Gilgenbach, Y. Y. Lau, and R. A. Bosch' \\ Nuclear Engineering Department, Intense Energy Beam Interaction Laboratory, University of Michigan, \\ Ann Arbor, Michigan 48109-2104
}

(Received 19 August 1993; accepted for publication 26 October 1993)

\begin{abstract}
The results of an experimental program whose sole objective is to investigate the cumulative beam breakup instability (BBU) in electron beam accelerators are presented. The BBU growth rate scalings are examined with regard to beam current, focusing field, cavity $Q$, and propagation distance. A microwave cavity array was designed and fabricated to excite and measure the cumulative BBU resulting from beam interactions with the deflecting $\mathbf{T M}_{110}$ cavity mode. One phase of this experiment used high $Q(\approx 1000)$ cavities with relatively large frequency spread $\left(\Delta f / f_{0} \approx 0.1 \%\right)$. The observed $\mathrm{TM}_{110}$ mode microwave growth between an upstream (second) and a downstream (tenth) cavity indicated BBU growth of $26 \mathrm{~dB}$ for an electron beam of kinetic energy of $750 \mathrm{keV}, 45 \mathrm{~A}$, and focused by a $1.1 \mathrm{kG}$ solenoidal field. At beam currents of less than $100 \mathrm{~A}$ the experiments agreed well with a two-dimensional continuum theory; the agreement was worse at higher beam currents $(>100 \mathrm{~A})$ due to beam loading. The second-phase experiments used lower $Q(\approx 200)$ cavities with relatively low frequency spread $\left(\Delta f / f_{0} \approx 0.03 \%\right)$. Theory and experiment agreed well for beam currents up to $220 \mathrm{~A}$. Distance scaling experiments were also performed by doubling the propagation length. Instability growth reduction experiments using the technique of external cavity coupling resulted in a factor of four decrease in energy in BBU growth when seven internal beam cavities were coupled by microwave cable to seven identical external dummy cavities. A theory invoking power sharing between the internal beam cavities and the external dummy cavities was used to explain the experimental reduction with excellent agreement using an equivalent circuit model.
\end{abstract}

\section{INTRODUCTION}

The beam breakup (BBU) instability is one of the most serious of the electron beam instabilities that arise in linear accelerators. ${ }^{1}$ This instability results from the coupling of transverse beam oscillations and nonaxially symmetric electromagnetic modes of the accelerating structure. The BBU instability can be classified into two major categories, regenerative and cumulative. In the regenerative BBU, upstream (backward) propagation of the nonaxially symmetric mode provides feedback for amplification within a single accelerator section. ${ }^{2}$ In the cumulative (also called multisection) BBU instability, the nonaxially symmetric modes of different accelerator sections are coupled only by the passage of the electron beam. The study presented in this paper will deal exclusively with the cumulative BBU. The BBU is capable of growing over time in one cavity as the trailing portions of the beam become more severely deflected than the proceeding portions. This instability can also grow over distance as the amplifying disturbances are carried along the beam, resulting in a range of macroscopic effects, from simple degradation of beam quality (emittance growth) to total loss of beam current if the beam strikes the cavity walls. BBU therefore places a limit on the beam's pulse length, propagation distance, and the beam current.

\footnotetext{
${ }^{a)}$ Present address: Sandia National Laboratories, Dept. 1231, Albuquerque, NM 87185.

b) Present address: Synchrotron Radiation Center, University of Wisconsin-Madison, Stoughton, WI 53589-3097.
}

Despite the serious limitations imposed on accelerators by the BBU, there have been few publications where BBU is systematically studied in experiments and compared with the theories. The goal of the experiments presented in this paper is to systematically study the experimental scaling laws and growth rates for the BBU instability. With this goal in mind, the experimenter is free to investigate parameters that have limited variability in large scale accelerator programs. These parameters include beam current, focusing magnetic field, pulse lengths, cavity $Q$, and BBU frequency. This work then is the first experiment designed whose sole objective is to investigate the behavior of the BBU. A summary of some of these University of Michigan experiments can be found in Ref. 3. A second objective is to develop novel techniques to reduce the growth of the BBU. One such technique performed during the course of this research is the demonstrated use of "external cavity coupling.",4

The experimental approach proceeded in three phases. The first experiments examined the growth of the BBU using an approximately $1 \mathrm{~m}$ long array of $\mathrm{rf}$ resonant pillbox cavities characterized by relatively high $Q(\approx 1000)$ and moderately large frequency spread $\left(\Delta f / f_{0} \approx 0.1 \%\right)$ among the cavities. These high $Q$ experiments demonstrate the importance of beam loading. To reduce the effects of beam loading, in the next phase, a cavity array with a lower $Q$ was employed ( $\approx 200$ ), but the frequency spread was much smaller $\left(\Delta f / f_{0} \approx 0.03 \%\right)$. Two subsets of these latter experiments were performed, investigating BBU behavior over beam propagation distances of 1 and $2 \mathrm{~m}$. The 


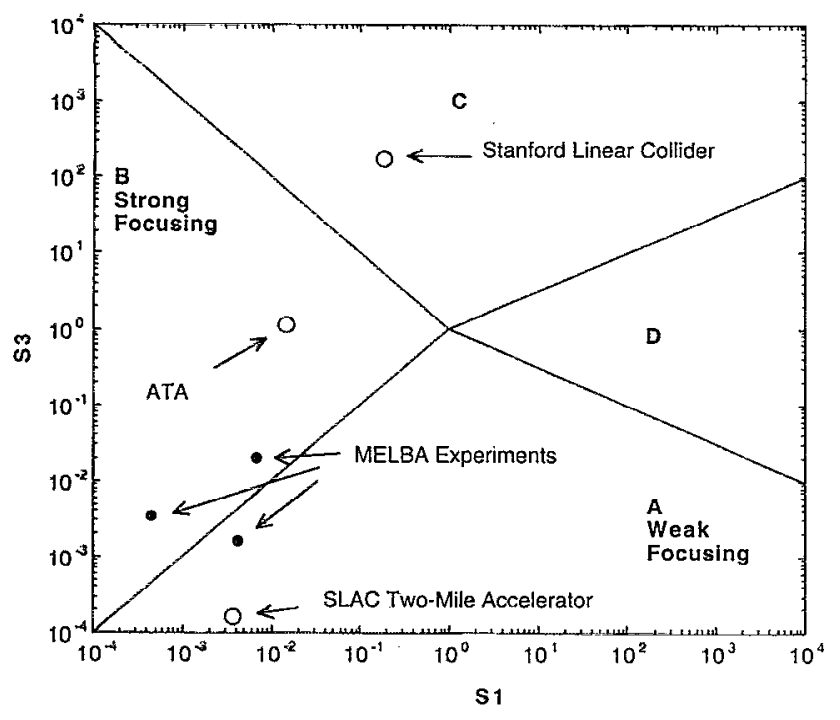

FIG. 1. Comparison of MELBA to other well-known accelerators in BBU growth rate parameter space (from Ref. 7).

parameters for these experiments place the BBU growth rate scaling close to the boundary between the weak ${ }^{1}$ and strong focusing ${ }^{5,6}$ regimes (defined in Ref. 7). The third phase consisted of experiments designed to reduce the BBU growth rate using the aforementioned external cavity coupling.

The electron beam accelerator used in this research is the Michigan Electron Long Beam Accelerator (MELBA).$^{8}$ A useful comparison of MELBA growth rate scalings to those of other well-known accelerators is shown in Fig. 1. As explained in Ref. 7, the parameter $S 1$ is proportional to beam current, and inversely proportional to the square of the applied focusing field, and $S 3$ is the ratio of accelerator length normalized by the betatron wavelength to the pulse length normalized by the period of the deflecting mode. Each region on the graph represents a unique BBU growth rate scaling defined in Ref. 7.

\section{HIGH $Q$ LARGE FREQUENCY SPREAD EXPERIMENTS}

The MELBA diode operates with the following parameters: voltage $=-0.7$ to $-0.8 \mathrm{MV}$, diode current $=1-10$ $\mathrm{kA}$, and pulse length $=0.3-5 \mu \mathrm{s}$, with flattop voltage provided by an Abramyan-type compensation stage over $1 \mu \mathrm{s}$.

The experimental configuration is shown in Fig. 2. The electron beam is generated from an explosive emission velvet cathode. This cathode consists of a glyptal-coated hemispherical-end cathode stalk with a velvet emitting button attached to the end of the cathode stalk. This cathode is advantageous for its slow diode closure, limited edge emission, and relatively long glyptal life. The anode is a graphite plate ( $1 / 8$ in. thick) located $10.8 \mathrm{~cm}$ from the end of the cathode. A circular aperture with a diameter of $2 \mathrm{~cm}$ is centered on the anode plate to extract $40-300 \mathrm{~A}$ into the transport chamber.

The diode chamber is immersed in a solenoidal magnetic field that can be varied from 0.5 to $1.2 \mathrm{kG}$. The

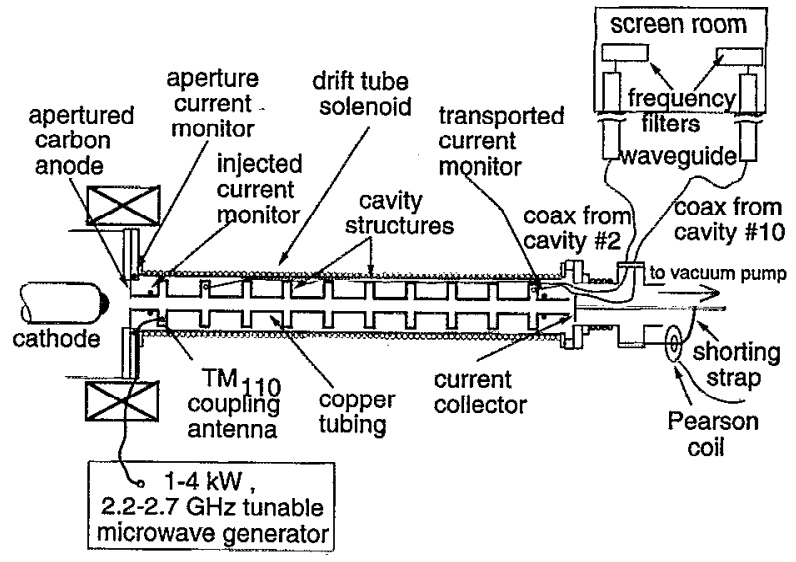

FIG. 2. Experimental configuration.

transport chamber is composed of a $1 \mathrm{~m}$ long drift tube surrounded by solenoid coils pulsed independently from the diode. The current to the solenoid is provided by a two-stage, double-polarity, electrolytic capacitor bank that produces a magnetic field up to $3.5 \mathrm{kG}$. Since the diode coils and the drift tube solenoid overlap, the superposition of the solenoidal fields causes a total $B$-field profile that is not quite uniform in the experimental chamber. The profile is higher on the upstream end before tapering to a flattop about halfway down the drift tube.

Within the drift tube are ten brass pillbox resonant cavities separated by smaller diameter copper tubes. Each cavity has a radius of $6.9 \mathrm{~cm}$ and a length of $2.0 \mathrm{~cm}$. Within each cavity is a small loop antenna oriented to be sensitive to the $\mathrm{TM}_{110}$ cavity mode. With cylindrical pillbox cavities, the $\mathrm{TM}_{110}$ mode is the most significant BBU mode, because it is the fundamental, nonaxially symmetric TM mode, and it produces the maximum instability growth. The length of the separation tubes is $6.5 \mathrm{~cm}$, giving an intercavity distance of $8.5 \mathrm{~cm}$. The diameter of the tubes is $3.8 \mathrm{~cm}$, which is below the $\mathrm{TM}_{010}$ cutoff diameter, and thus serves to isolate each cavity from $\mathrm{rf}$ crosstalk. The measured attenuation of the $\approx 2.5 \mathrm{GHz}, \mathrm{TM}_{110}$ mode microwaves is $26 \mathrm{~dB}$ from cavity to adjacent cavity. This ensures that the BBU under study is the cumulative type (i.e., nonregenerative type).

The first cavity (closest to the anode) has its $\mathbf{T M}_{110}$ mode primed externally by an Epsco (model PG5KB) microwave pulse generator. The priming microwave pulse is generally $3 \mu$ s long and begins before the e-beam is present. The power of the injected microwaves is generally $1 \mathrm{~kW}$. The purpose of this procedure is to provide sufficient initial transverse modulation to the beam to allow BBU growth in a ten cavity system.

The general technique used in these experiments is to investigate the BBU instability by taking measurements on the characteristic microwaves produced by the instability. In particular the magnitude of BBU growth is determined through the growth of the $\mathrm{TM}_{110}$ microwave power between the second and tenth cavities.

In the second cavity the e-beam-induced of is measured 
TABLE I. Parameters for high $Q$, large frequency spread cavities.

\begin{tabular}{ccc}
\hline \hline $\begin{array}{c}\text { Cavity } \\
\text { No. }\end{array}$ & $\begin{array}{c}\mathrm{TM}_{110} \text { resonant } \\
\text { frequency } \\
(\mathrm{GHz})\end{array}$ & $\mathrm{TM}_{110} Q$ \\
\hline 1 & 2.5190 & 660 \\
2 & 2.5200 & 1090 \\
3 & 2.5200 & 1420 \\
4 & 2.5201 & 1800 \\
5 & 2.5265 & 530 \\
6 & 2.5270 & 680 \\
7 & 2.5230 & 1160 \\
8 & 2.5240 & 920 \\
9 & 2.5248 & 680 \\
10 & 2.5250 & 1010 \\
Average & $2.5230 \pm 0.1 \%$ & $1000 \pm 310$ \\
\hline \hline
\end{tabular}

via the loop antenna. The microwaves are propagated out of the experimental chamber through RG/0.405 U semirigid transmission cable to a vacuum feedthrough, to $\mathrm{RG} / 8$ cable, to an $S$-band waveguide. The waveguide runs to a Faraday cage where the signal is attenuated and filtered for frequency information. Part of the microwave signal is diverted into a filter that passes $2.523 \mathrm{GHz} \pm 11$ $\mathrm{MHz}$. This frequency, as noted above, corresponds to the $\mathrm{TM}_{110}$ BBU frequency.

The rf power in the tenth (last) cavity is measured in the same way with its own set of cables and waveguides. The growth of the BBU instability is determined by the decibels of growth in $2.5 \mathrm{GHz}$ microwave power between the second and tenth cavities.

Electron beam current is measured at several points in the transport experiment. Cathode stalk current in the diode is measured by a $B$-dot loop in the MELBA oil tank, behind the insulators. Extracted current is monitored by a Rogowski coil in the flange after the anode. Injected current is measured by a Rogowski coil before the first cavity and exit current is detected by a Rogowski coil after the last cavity. Exiting current is also measured by a carbon plate/current collector which is grounded by a strap which passes through a Pearson current transformer.

The exact $\mathrm{TM}_{110}$ resonant characteristics of the ten cavities in the transport array are listed in Table I. The cavities are numbered in order of beam encounter. As indicated, the average resonant frequency is $2.523 \mathrm{GHz}$ $\pm 0.1 \%$, and the average $Q$ is $1000 \pm 310$. Comparison of the frequency spread $\left(\Delta f / f_{0}=0.1 \%\right)$ and average resonant linewidth $(1 / Q=0.1 \%)$ reveals that they are approximately equal, and thus the frequency spread among the cavities can be referred to as large.

Figure 3 shows a typical data set of electron beam and If signals from a high $Q$, large-frequency-spread BBU transport experiment. The uppermost signal trace (a) is the voltage applied to the diode by the MELBA Marx generator. The voltage has a total length of about $700 \mathrm{~ns}$ and the duration of the flattop is about $400 \mathrm{~ns}$. The voltage magnitude at the flattop is $-750 \mathrm{kV}$. The second trace (b) is the signal from the Rogowski coil that encircles the entrance to the first cavity. This signal has a flattop amplitude

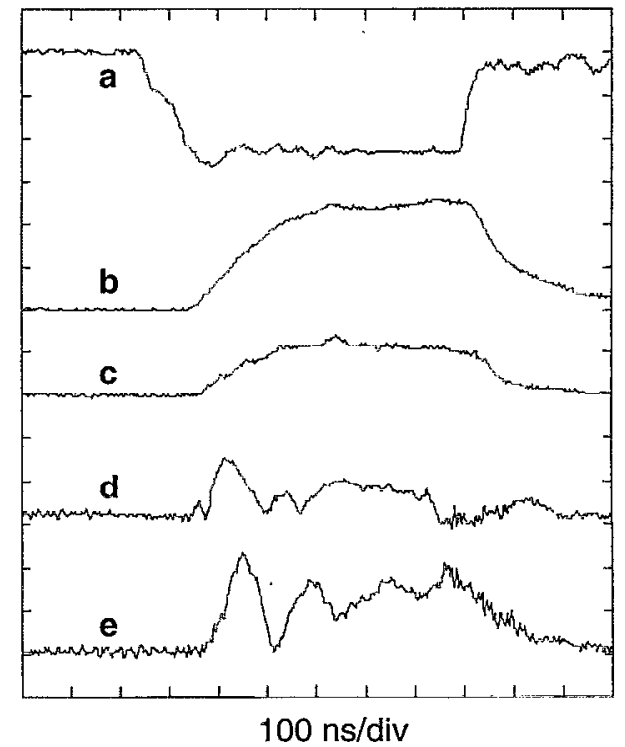

FIG. 3. Experimental data taken from two similar shots. (a) Diode voltage ( $310 \mathrm{kV} / \mathrm{div}$ ), flattop is $750 \mathrm{kV}$. (b) Injected current ( $92 \mathrm{~A} / \mathrm{div}$ ), maximum is $230 \mathrm{~A}$. (c) Transported current ( $40 \mathrm{~A} / \mathrm{div}$ ), flattop is $46 \mathrm{~A}$. (d) Detector signal of second cavity if ( $100 \mathrm{mV} / \mathrm{div})$, signal attenuated by $12 \mathrm{~dB}$. (e) Detector signal of tenth cavity $\mathrm{rf}(100 \mathrm{mV} / \mathrm{div})$, signal attenuated by $32 \mathrm{~dB}$. Time scale is $100 \mathrm{~ns} / \mathrm{div}$. Solenoidal magnetic field is $1.1 \mathrm{kG}$.

corresponding to approximately $220 \mathrm{~A}$. The center trace (c) represents the transported current. This trace is the signal from the Pearson coil that surrounds the cable which connects the collector plate to ground. The magnitude of beam current on the flattop for this signal is $46 \mathrm{~A}$. The drift tube solenoid produced a magnetic field of 1100 $\mathrm{G}$ for this particular shot. The fourth trace (d) is the diode crystal detector signal of the microwaves picked up by the loop antenna in the second cavity. This signal has passed through the frequency filter which passes frequencies at $2.523 \pm 0.011 \mathrm{GHz}$. The external attenuation added to the second-cavity microwaves is $12 \mathrm{~dB}$. The lowermost trace (e) is the microwave crystal detector signal as received by the loop antenna in the tenth (last) cavity; this signal has passed through a frequency filter similar to that used for the second-cavity diagnostics. The external attenuation added for this signal is $32 \mathrm{~dB}$. The external microwave priming source was tuned to the exact resonance of the first cavity (see Table I), $2.519 \mathrm{GHz}$, and the power of the priming pulse was approximately $1 \mathrm{~kW}$.

The experimental spatial growth rate for the BBU instability can be determined from the data presented in Fig. 3. The experimental growth rate is ascertained through examination of the rf signals [traces (d) and (e) from Fig. 3]. These traces are diode crystal detector signals which give measurements of microwave power injected into the detectors. Scrutiny of the microwaves from the second cavity [signal (d) of Fig. 3] shows a detector signal of about 80 $\mathrm{mV}$ along the flattop of the MELBA voltage pulse. A signal magnitude of $80 \mathrm{mV}$ corresponds to a power of $9 \mathrm{~mW}$ for this particular detector. Before entering the detector, the signal was purposely reduced by $12 \mathrm{~dB}$ in external 
attenuation and also by another $2.7 \mathrm{~dB}$ from resistive and insertion losses in the cable, waveguide, and frequency filter. Thus, the total power of the $\mathbf{T M}_{110}$ microwaves on the flattop picked up by the loop antenna in the second cavity is $270 \mathrm{~mW}$.

The $\mathrm{TM}_{110}$ microwave signal in the tenth cavity has a magnitude of about $160 \mathrm{mV}$ on the flattop. Note that the overall shape of the tenth-cavity microwave power is similar to that of the second cavity. There is about $40 \mathrm{~ns}$ of delay between the tenth and second cavity signals due to a longer waveguide used to transport the microwaves from the tenth cavity. This suggests that the magnitude of the microwaves in the tenth cavity is a direct result of growth from the second-cavity microwaves. This detector's calibration at $160 \mathrm{mV}$ gives a detector power of $35 \mathrm{~mW}$. The added attenuation in this signal is $32 \mathrm{~dB}$ with an additional reduction of $2.9 \mathrm{~dB}$ from resistive and insertion losses. Thus, the total power received by the antenna in the tenth cavity is $99 \mathrm{~W}$. Therefore, the growth in $\mathrm{TM}_{110}$ microwave power between the second and tenth cavities as sampled by the loop antennas is $99 \mathrm{~W} / 0.27 \mathrm{~W} \approx 370$, which is equivalent to $26 \mathrm{~dB}$. The distance between the antennas is $68 \mathrm{~cm}$, giving a spatial microwave growth rate of $38 \mathrm{~dB} / \mathrm{m}$.

A theoretical prediction for the amount of BBU growth can be found using the continuum dispersion relation ${ }^{7,9,10}$ for solenoidal focusing: ${ }^{11}$

$$
\left(\Omega^{2}-\omega_{c} \Omega+\Gamma\right)\left(\Omega^{2}+\omega_{c} \Omega+\Gamma\right)=0,
$$

where $\Omega=\omega-k v$ and

$$
\Gamma=\frac{2 \omega_{0}^{4} \epsilon}{-\omega^{2}+\omega_{0}^{2}+i \omega \omega_{0} / Q} .
$$

The variable $k$ is the wave number of the instability, $\omega$ is the frequency which undergoes BBU growth, $\omega_{0}$ is the $\mathrm{TM}_{110}$ angular resonant frequency, $v$ is the electron beam velocity, $Q$ is the cavity quality factor, $\omega_{c}$ is the relativistic angular betatron frequency, and $\epsilon$ is the dimensionless coupling constant, ${ }^{7}$ where

$$
\epsilon=0.422 \frac{l}{L} \frac{I}{17 k A} \frac{\beta}{\gamma}
$$

Here, $l$ is the cavity length, $L$ is the spacing of cavity centers, $I$ is the e-beam current, and $\beta$ and $\gamma$ have their usual meanings. For the experimental parameters of $l=2$ $\mathrm{cm}, L=8.5 \mathrm{~cm}$, transported current $=46 \mathrm{~A}$, and kinetic energy $=750 \mathrm{keV}$, one finds $\epsilon=1.0 \times 10^{-4}$. A solenoidal field of $1.1 \mathrm{kG}$ yields $\omega_{c}=7.85 \mathrm{Grad} / \mathrm{s}$, the BBU frequency is the priming frequency, $\omega=2 \pi \times 2.519 \mathrm{GHz}$, and the average $\mathrm{TM}_{110}$ frequency is $\omega_{0}=2 \pi \times 2.523 \mathrm{GHz}$. Insertion of these values into Eq. (1) produces a theoretical spatial growth rate in the continuum model of

$$
\operatorname{Im}(k)=4.90 \mathrm{~m}^{-1} \text {. }
$$

Thus, the e-folding length for the instability is $20.4 \mathrm{~cm}$. This implies a if amplitude growth over the $68 \mathrm{~cm}$ between the second and the tenth cavities of $\exp \{68.0 / 20.4\}=28.0$. An amplitude growth of 28 translates to $29 \mathrm{~dB}$ gain. Thus, the two-dimensional continuum theory predicts $29 \mathrm{~dB}$ of

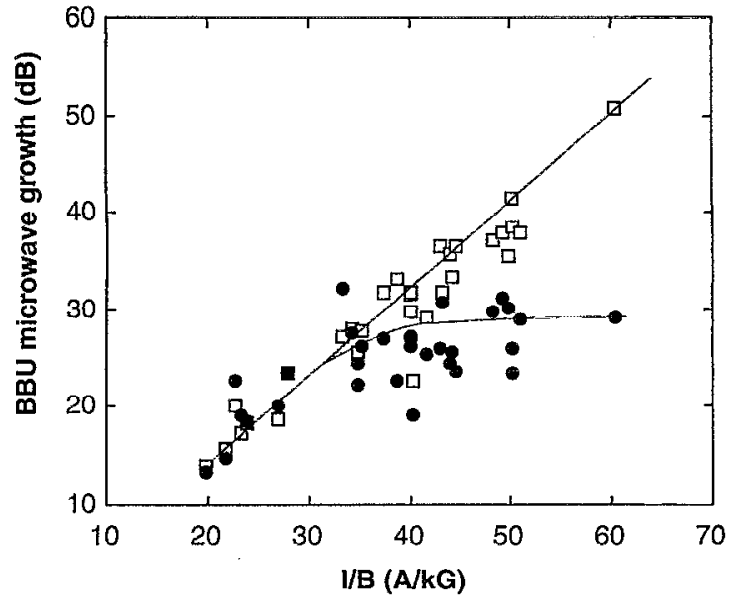

FIG. 4. Graph of beam breakup instability growth vs the ratio of transported current to magnetic field, $I / B$. For each point representing experimentally measured growth (closed circles), the corresponding theoretical prediction for growth using the same beam parameters is also plotted (open squares). The lines are guides for the eye.

BBU growth for a $750 \mathrm{keV}$ electron beam with current $=46 \mathrm{~A}$, and a solenoidal focusing field of $1.1 \mathrm{kG}$. This is in reasonably good agreement with the experimental growth of $26 \mathrm{~dB}$, considering the use of a continuous model for the few cavity system and the neglect of beam loading and frequency detuning among the cavities.

The preceding continuum model treats the transverse impulsive forces from the accelerating cavities as a continuous force per unit length. This approximation limits the dispersion relations to the cases where instability scale lengths (i.e., e-folding length, and wavelength of the BBU disturbance) are long compared to the cavity spacing. Modeling the transverse force on the beam resulting from pillbox cavities placed at discrete locations produces the following dispersion relation: ${ }^{11}$

$$
\cos \left(\frac{L \Omega}{v} \pm \frac{L \omega_{c}}{2 v}\right)=\cos \frac{L \omega_{c}}{2 v}+\frac{\Gamma L}{\omega_{c} v} \sin \frac{L \omega_{c}}{2 v} .
$$

The discrete cavity dispersion relation of Eq. (2) yields a prediction of $24 \mathrm{~dB}$ growth in the BBU, when the effect of frequency spread is taken into account, i.e., the growth in each cavity is computed locally using the individual parameters for each cavity (see Table $\mathrm{I}$ ).

An unfortunate circumstance in this experiment is the fact that in order to increase the amount of transported current, the applied magnetic field must also be increased. Therefore, it is difficult to determine the scaling of the BBU growth versus current while holding the magnitude of the focusing field constant or vice versa. An attempt to incorporate the $I$ vs $B$ dependence into the growth rate analysis examines the BBU growth rate versus the ratio of current to magnetic field, $I / B$. Figure 4 plots the experimental and theoretical growth of the BBU vs $I / B$ using the two-dimensional continuum theory, Eq. (1).

In Fig. 4, it is evident that the BBU growth predicted by continuum theory (open squares) agrees well with BBU growth in experiments with low $I / B$. For large values of 


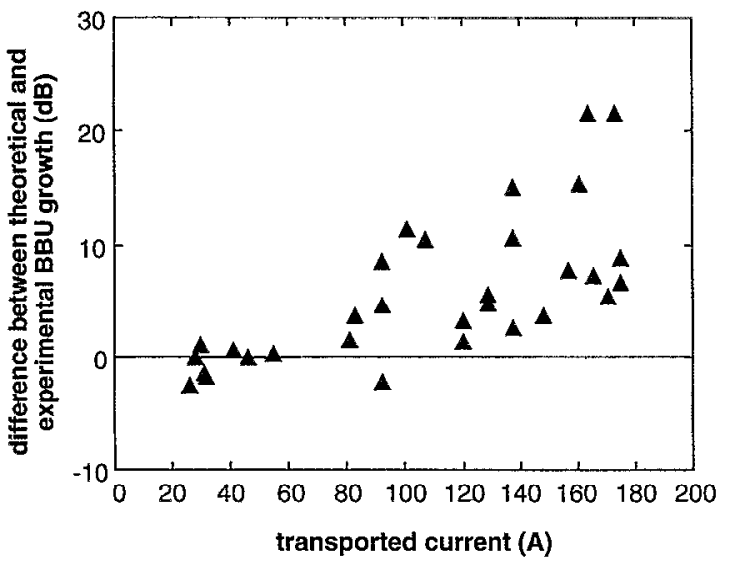

FIG. 5. Graph showing the difference between theoretically computed and experimentally observed BBU growth (i.e., $\Gamma_{\text {theory }}-\Gamma_{\text {experiment }}$ ) vs transported beam current. Horizontal line shows position of exact agreement.

$I / B$, however, there is significant divergence between theory and experiment. Application of the discrete cavity theory, Eq. (2), does not provide much better agreement. An explanation for this difference between theory and observation could be "beam loading," which changes the electromagnetic properties of a cavity by the presence of an e-beam. One effect of beam loading is to shift the resonant frequency for a beam-filled cavity according to ${ }^{12}$

$$
\omega_{0}^{\prime}=\sqrt{\omega_{0}^{2}+\omega_{\mathrm{pe}}^{2}},
$$

where $\omega_{0}^{\prime}$ is the corrected upshifted resonant frequency, $\omega_{0}$ is the angular resonant frequency of the cold cavity, and $\omega_{\text {pe }}$ is the angular plasma frequency of the electron beam given by

$$
\omega_{\mathrm{pe}}^{2}=\frac{4 I_{b} c^{2}}{r_{w}^{2}(17 \mathrm{kA})} \sqrt{\frac{1+\alpha^{2}}{\gamma^{2}-1}}
$$

where $I_{b}$ is the beam current, $r_{w}$ is the radius of the cavity, and $\alpha$ is the perpendicular to parallel electron velocity ratio. For example, an electron beam with parameters: $I_{b}=100 \mathrm{~A}, V=750 \mathrm{kV}, r_{w}=6.9 \mathrm{~cm}$, and $\alpha=0.1$, and $\omega_{0}=2 \pi \times 2.523 \mathrm{GHz}$, the corrected angular resonant frequency is $\omega_{0}^{\prime}=2 \pi \times 2.524 \mathrm{GHz}$. This increase may seem slight, but it is important to remember that the cavities have a high $Q$ (narrow linewidth). For $I_{b}=128$ A the resonance of the average cavity is shifted $0.05 \%$. This shift is equal to the linewidth of a cavity with $Q=1000$.

In other words, if the current is high enough, then the beam can "detune" the cavities with respect to the beam modulation-cavity resonance condition which drives the BBU. The effect of this shift is that the frequency of the BBU disturbance (equal to the priming frequency) is too low to drive the (detuned) $\mathrm{TM}_{110}$ mode, leading to the observed reduction of BBU growth in the experiments.

Figure 5 shows the difference between the predicted magnitude of BBU growth from continuum theory and the observed growth ( $\left.\Gamma_{\text {theory }}-\Gamma_{\text {experiment }}\right)$ versus transported current. The larger discrepancy at higher beam current is strong evidence for the beam loading effect. It is also interesting to note that significant difference appears to begin at approximately $100 \mathrm{~A}$, which is where the frequency upshift is comparable to the linewidth of the average cavity.

In order to gain further understanding of the detuning phenomenon and its effect on the BBU growth rate, experiments in which the priming frequency was changed were carried out. These experiments could be considered as the converse of beam loading. Beam loading causes the resonant frequencies of the cavities to be detuned with respect to the frequency of the beam modulation. Changing the priming frequency, however, causes the beam modulation to be altered with respect to the cavity resonant frequencies. For these experiments the beam current is kept low ( $<50 \mathrm{~A}$ ) so that the beam loading effect is slight. An advantage in this case is that the priming frequency can be very accurately determined, whereas detuning through beam loading is ambiguous because of uncertainties in the exact frequency upshift due to the beam only partially filling the cavity, and differences in $Q$ 's and variations in resonant frequencies among the cavities.

When the priming microwaves are turned off, very little signal is observed on the crystal detectors, indicating undetectable instability growth arising from noise over the ten cavities. This suggests that the frequency of modulation applied to the beam through priming will be the dominant frequency available for BBU growth. In other words, the effect of priming is so strong that growth at any any other frequency will be small compared to the BBU growth of the priming microwaves. This feature has the additional advantage of observing BBU growth at a frequency other than the exact resonant frequency.

A summary of microwave growth for several shots taken over a range of priming frequencies is shown in Fig. 6. The currents for these data range from 40 to $50 \mathrm{~A}$ and the applied magnetic field for all shots is $1.1 \mathrm{kG}$. Superimposed on the data are the dispersion relations, $\operatorname{Im}(k)$, from the continuum theory Eq. (1) (thin lined curve), and the discrete cavity theory, Eq. (2) (thick lined curve), with $\omega$ equal to the priming frequency. The continuum theory uses $\omega_{0}$ equal to the average cavity $\mathbf{T M}_{110}$ resonant frequency of $2.523 \mathrm{GHz}$ and thus neglects frequency spread. The discrete cavity theory is the sum of predicted growths resulting from each cavity using each cavity's own $\omega_{0}$ and $Q$. The peaking of the data near the average $\mathrm{TM}_{110}$ resonant frequency is evidence that the BBU instability is indeed responsible for the observed microwave growth. The data also trace a broader curve than the continuum theory. This feature is due to the resonant frequency spread among the cavities in the array. The initial cavity's $\mathbf{T M}_{110}$ resonant frequency is $2.519 \mathrm{GHz}$. Thus, the $\mathrm{TM}_{110}$ fields are strongest and produce the most initial beam modulation when the priming is tuned to $2.519 \mathrm{GHz}$. In the region of 2.519 $2.524 \mathrm{GHz}$, both the data and the discrete cavity theory indicate nearly constant growth rate. Intuitively, this constant region exists because near $2.519 \mathrm{GHz}$, cavities $1-4$ should show near maximum growth, but cavities 5-10 should exhibit decreased growth. At $2.524 \mathrm{GHz}$ the situation is reversed. Beyond $2.524 \mathrm{GHz}$ the microwave growth 


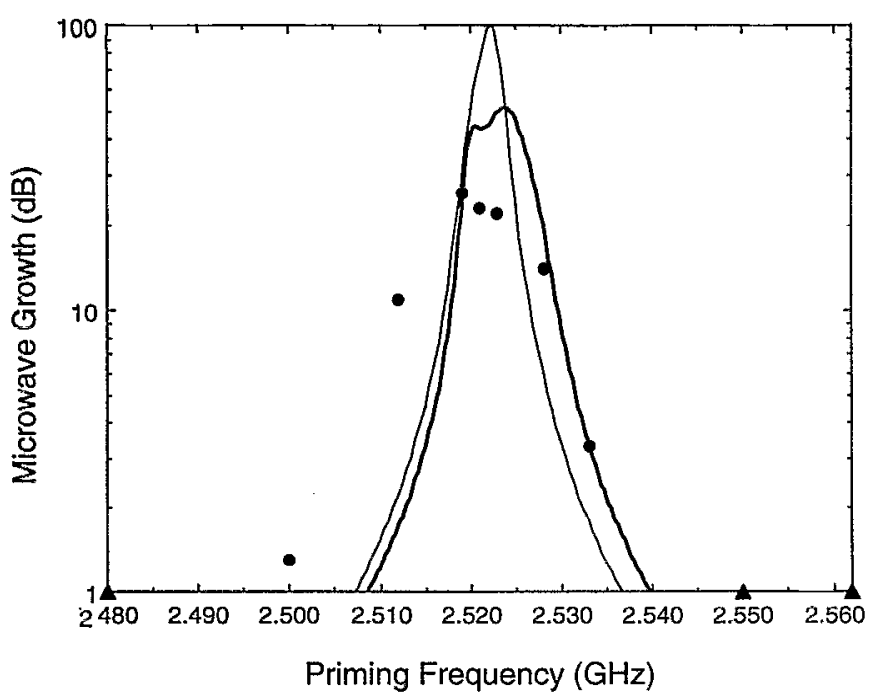

FIG. 6. Microwave growth vs priming frequency. Experimentally observed microwave growth (closed circles) is shown with the theoretical growth curves for $I_{b}=45 \mathrm{~A}$ and $B=1.1 \mathrm{kG}$. The thick lined curve is the predicted growth from the discrete cavity theory [Eq. (2)], and the thin lined curve uses the continuum theory which does not include frequency spread. The closed triangles along the abscissa represent experimental growth below the level of detectability.

diminishes rapidly following the theoretical curves.

The detuning experiments show that the coupling between the beam modulation and cavity. fields is strongly dependent upon the resonant condition. The theory has proven accurate at low currents, but at high currents the experiments are not adequately explained by the existing theory partly because of beam loading and partly because of the significant spread in the resonant frequencies. Some obvious improvements to this situation are apparent. One improvement would be to accurately match $(<0.1 \%)$ the $\mathrm{TM}_{110}$ resonant frequencies. Especially important would be to match the frequency of the initial priming cavity to the average resonant frequency. This motivated our lowering of the $Q$ 's of the cavities, the result of which is reported next.

\section{LOW $Q$, SMALL FREQUENCY SPREAD EXPERIMENTS}

In an attempt to create a fair test between theory and experiment that would be valid at high e-beam currents, the cavity array was modified to a lower average $Q$, and a lower frequency spread. Table II lists the $\mathrm{TM}_{110}$ mode parameters for this second array. The $Q$ lowering was achieved by inserting a thin annulus of microwave absorber (Eccosorb) into each cavity.

Low $Q$ experiments using both 10 and 19 cavities have been performed. The BBU growth results with ten cavities have been published elsewhere (see Ref. 3), and they are included on the right-hand side of Fig. 7 for later comparison. Note that there is no significant deviation from theoretical growth at high values of $I / B$, indicating mitigation of the beam loading effect.
TABLE II. Parameters for low $Q$, small frequency spread cavities.

\begin{tabular}{ccc}
\hline \hline $\begin{array}{c}\text { Cavity } \\
\text { No. }\end{array}$ & $\begin{array}{c}\mathrm{TM}_{110} \text { resonant } \\
\text { frequency } \\
(\mathrm{GHz})\end{array}$ & $\mathrm{TM}_{110} Q$ \\
\hline 1 & 2.5070 & 230 \\
2 & 2.5070 & 290 \\
3 & 2.5060 & 215 \\
4 & 2.5082 & 220 \\
5 & 2.5070 & 280 \\
6 & 2.5072 & 230 \\
7 & 2.5076 & 160 \\
8 & 2.5078 & 185 \\
9 & 2.5085 & 180 \\
10 & 2.5086 & 170 \\
Average & $2.5075 \pm 0.03 \%$ & $215 \pm 45$ \\
\hline \hline
\end{tabular}

Experiments using an additional 9 cavities, for a total of 19 , have been recently performed. A longer $(2 \mathrm{~m})$ solenoidal drift tube was employed, but otherwise the configuration and diagnostics are similar to those of the 10 cavity experiments (shown in Fig. 2). The parameters for the additional nine cavities (11-19) are shown in Table III.

The left-hand portion of Fig. 7 illustrates the BBU growth versus $I / B$ for the 19-cavity system. The lower $I / B$ values for the 19-cavity experiment are due to the lower magnetic field and thus lower transported current capable of being generated by the $2 \mathrm{~m}$ solenoid. For each experimental datum the corresponding discrete-cavity theoretical growth is also plotted. Note that the slope of the growth for the 10-cavity case is approximately half of that for the 19-cavity case, as expected.

Experimental data were also taken to gain information about the dispersion relation when $\omega \neq \omega_{0}$ for the 10-cavity case and is shown in Fig. 8. Just as in the high $Q$, large frequency spread experiments, the priming frequency was varied about the resonant frequency. This set of experi-

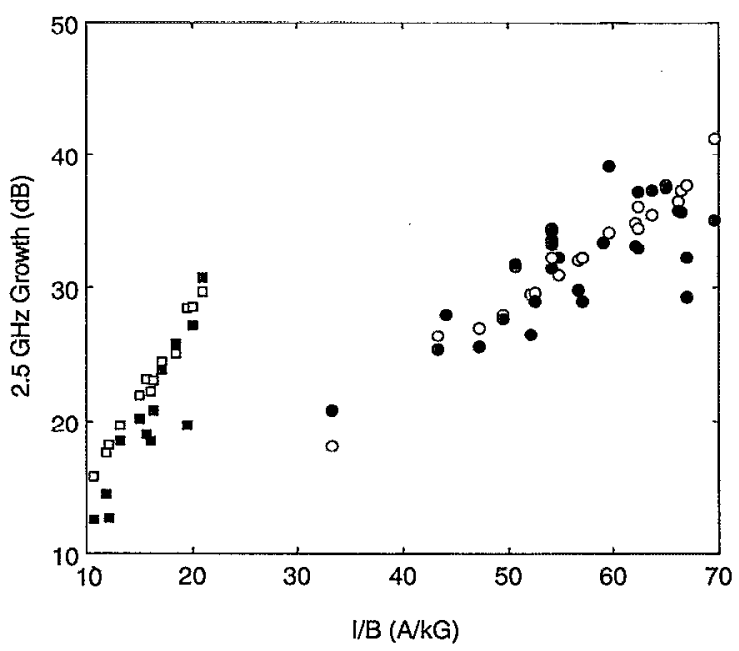

FIG. 7. BBU growth (dB) vs ratio of beam current to magnetic field, $I / B$ $(\mathrm{A} / \mathrm{kG})$. Experimental data (closed symbols) are plotted with corresponding theoretical growth (open symbols). Two experimental cases are shown: 19 cavities (squares) and 10 cavities (circles). 
TABLE III. Parameters for additional cavities in the 19 cavity experiment.

\begin{tabular}{ccc}
\hline \hline $\begin{array}{c}\text { Cavity } \\
\text { No. }\end{array}$ & $\begin{array}{c}\mathrm{TM}_{110} \text { resonant } \\
\text { frequency } \\
(\mathrm{GHz})\end{array}$ & $\mathrm{TM}_{110} \mathcal{Q}$ \\
\hline 11 & 2.5070 & 290 \\
12 & 2.5079 & 250 \\
13 & 2.5070 & 260 \\
14 & 2.5073 & 300 \\
15 & 2.5075 & 230 \\
16 & 2.5076 & 190 \\
17 & 2.5082 & 160 \\
18 & 2.5085 & 300 \\
19 & 2.5080 & 270 \\
19 cavity & $2.5076 \pm 0.03 \%$ & $230 \pm 45$ \\
average & & \\
\hline \hline
\end{tabular}

ments was also presented in Ref. 3, but is reproduced here for a ready comparison with Fig. 6 (high $Q$ experimental results). The plotted points (filled circles) are experimental data taken at the same magnetic field $(3.4 \mathrm{kG})$ and nearly the same e-beam current (190-215 A). The thicklined curve is the BBU growth as predicted by the discretecavity Eq. (2) using $I=210 \mathrm{~A}$, and using each cavity's specific resonant frequency and $Q$. The thin-lined curve is the continuum BBU growth Eq. (1) for the same parameters, but uses the average $\omega_{0}$ of $2 \pi \times 2.5075 \mathrm{GHz}$ and the average $Q$ of 215 . Note that the data follow the discrete cavity theory more closely in this case than in Fig. 6 .

\section{BBU REDUCTION USING EXTERNAL CAVITY COUPLING}

\section{A. Experimental results}

The purpose of the experimental program described in this paper is twofold. The first objective of this research was to successfully describe the behavior of the BBU in-

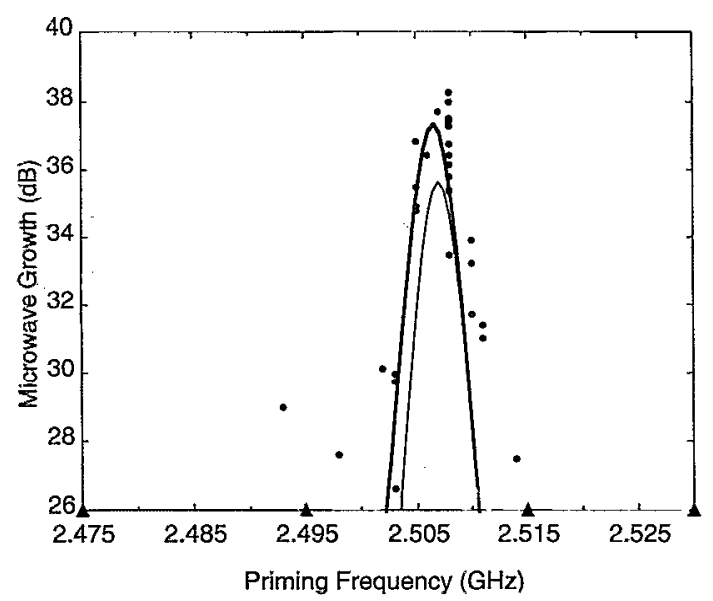

FIG. 8. Microwave growth dependence on the frequency of priming microwaves. Experimental data are represented by the closed circles. The thicker curve is the theoretical growth predicted by the discrete-cavity theory. The thinner curve is the theoretical growth predicted by the continuum theory. The triangles along the abscissa indicate experimental growth below the level of detectability (from Ref. 3 ).

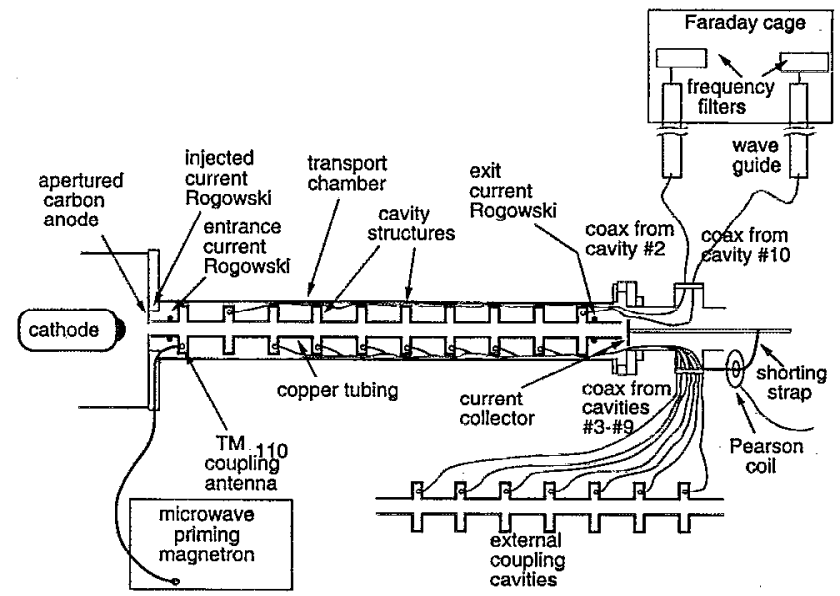

FIG. 9. External coupled cavity configuration.

stability by building an experiment specifically designed to study the BBU growth in a controlled manner. The results of the 10-cavity low $Q$, small frequency spread experiments detailed above and in Ref. 3 provide a good basis for pursuit of the next goal. The second intent of these experiments is to develop novel techniques for suppression of the BBU. In the high $Q$, large frequency spread case, BBU was reduced by beam loading and by breakup mode frequency variations. The knowledge that these effects can reduce BBU growth rates is not particularly novel. The use of if cures, especially by stagger tuning, has been studied elsewhere, ${ }^{2,10,13-16}$ although it was beneficial to confirm BBU growth reduction through these mechanisms.

A notably novel mechanism for BBU reduction has been suggested theoretically by Colombant, Lau, and Chernin ${ }^{17}$ and is particularly capable of being tested by the MELBA experiments. This method has been termed "external cavity coupling," and the initial experimental results using this method are detailed in Ref. 4 . In this method the main beam cavities are coupled by transmission line to identical external dummy cavities on the outside of the transport structure. The idea here is that the beam cavities are capable of "sharing" deflecting mode energy with the dummy cavities and thus reduce the magnitude of the $\mathrm{TM}_{110}$ mode fields in the beam cavities, with subsequent reduction in the BBU growth rate. ${ }^{18,19}$ The 10-cavity MELBA experimental configuration is readily adaptable to this method, since each cavity contains a coupling loop antenna to sample the $\mathrm{TM}_{110}$ microwaves. Each antenna can be connected to a coaxial cable to transmit the microwaves out of the cavity. In this way, the loop antennas can double as a microwave sampler and as a device to deliver the $\mathrm{TM}_{110}$ mode energy to a dummy cavity containing a similar coupling loop.

Figure 9 shows the experimental configuration used for the external cavity coupling experiments. This arrangement is almost identical to the configuration used for the low $Q$, small frequency spread baseline growth experiments described in Sec. III. The difference is that the seven intermediate cavities (3-9) between the second and tenth have 


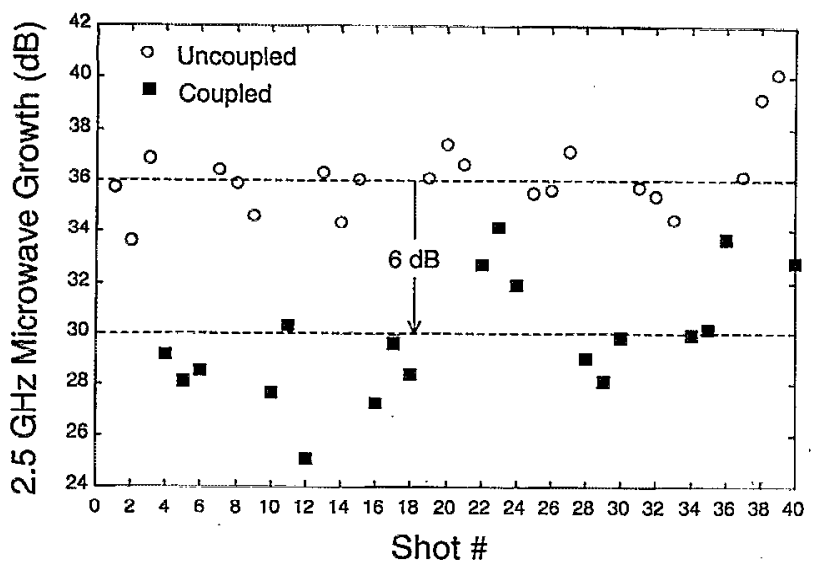

FIG. 10. Growth (dB) of the BBU microwave power for 40 different electron beam pulses showing uncoupled shots (open circles) and externally coupled shots (closed squares) (from Ref. 4).

their coupling loops connected via microwave cable to seven nearly identical cavities located externally to the solenoid drift tube. The length of this cable was chosen to be 16 wavelengths long. Typically an experimental run alternated between a few shots with the internal and external cavities coupled (loop antennas connected), followed by a few shots with the internal and external cavities uncoupled. When the cavities are uncoupled, the experiment is equivalent to the baseline low $Q$, large frequency spread $\mathrm{BBU}$ growth experiments described above.

A summary of BBU microwave growth data from some 40 shots (from Ref. 4) is presented in Fig. 10. The data show a consistent reduction of BBU growth from an average of $36 \mathrm{~dB}(\sigma= \pm 1.5 \mathrm{~dB}$ ) for the uncoupled case to an average of $30 \mathrm{~dB}(\sigma= \pm 2.4 \mathrm{~dB})$ for the coupled-cavity case. Thus, an average reduction in the BBU of $6 \mathrm{~dB}$ is measured for this system in which seven internal beam cavities are coupled to a nearly identical set of seven external dummy cavities. ${ }^{4}$

\section{B. Coupling constant, $\kappa$, determination}

Previous analysis ${ }^{4}$ has shown that the BBU spatial growth rate, $\Gamma$ (where total BBU growth is given by $e^{\Gamma z}$ ), is modified by a factor of $1 /\left(1+\kappa^{2} Q^{2}\right)$ to account for cavity coupling through a simple one-dimensional mutual inductance model. ${ }^{17-19}$ Thus, the external coupled cavity growth rate is $\Gamma /\left(1+\kappa^{2} Q^{2}\right)$. Here, $\kappa$ is the coupling constant and $Q$ is the cavity quality. This factor of $\kappa^{2} Q^{2}$ represents the ratio of power leaked to the dummy (external) cavity to the power remaining in the main (internal) cavity. ${ }^{4} \mathrm{~A}$ cold test was performed on a network analyzer (HP-8510) using two model cavities each with two coupling antennas. One antenna in each cavity was used to inject the microwaves and the second was used to transmit the rf power out of the cavity. This cold test experiment indicated that the power sharing ratio for this arrangement is $\kappa^{2} Q^{2}=0.13$. Using this value in the reduction factor yields an expected experimental reduction to $36 \mathrm{~dB}(1 /$ $1.13) \approx 32 \mathrm{~dB}$.

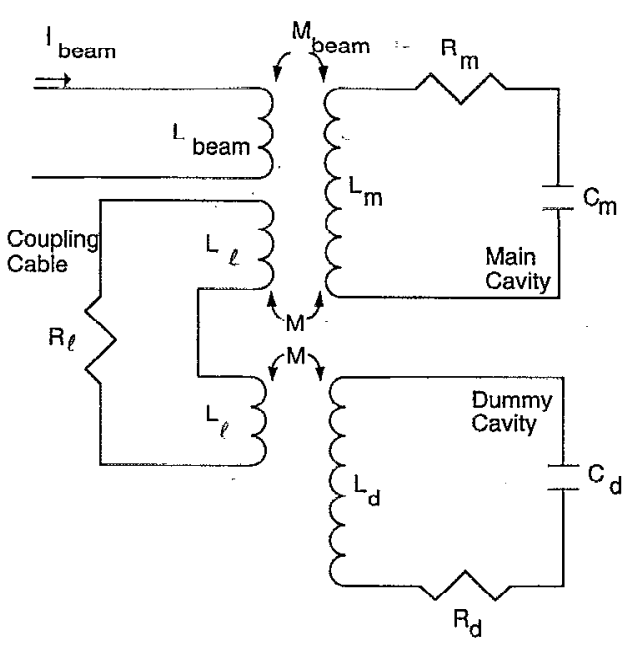

FIG. 11. Equivalent circuit representing the coupled cavities in the actual experimental configuration.

However, the cold test of the power sharing ratio differs from the actual experimental configuration. In the experiment, each cavity has only one coupling loop, thus the cold test may underestimate the magnitude of power sharing since the extra antennas provide additional inductance to the overall circuit. An alternative method to determine $\kappa$ has been developed using an equivalent circuit model similar to those used in coupled cavity klystron analyses. ${ }^{20}$ The equivalent circuit representing the experimental configuration is shown in Fig. 11. The critical parameter that governs the magnitude of power sharing is the mutual inductance, $M$, connecting the loop antenna circuits to the cavity circuits. The mutual inductance can be found from the formula: $:^{21,22}$

$$
\frac{\omega_{0}^{2} M^{2}}{R}=\frac{2 s^{2} J_{1}^{2}(3.83 r / b) Q}{J_{0}^{2}(3.83) \epsilon_{0} r^{2} \pi b^{2} l Z_{0} \omega_{0}},
$$

where $\omega_{0}$ is the angular TM $\mathrm{TM}_{110}$ resonant frequency, $R$ is the resistance assigned to the cavity circuit, $s$ is the area of the coupling loop, $r$ is the radial position of the antenna in the cavity, $b$ is the radius of the cavity, $l$ is the cavity length, and $Z_{0}$ is the characteristic impedance of the coupling cable. Table IV lists the circuit parameters shown in Fig. 11. Solving for power in the main and dummy cavities with the circuit program SPICE ${ }^{23}$ yields a power sharing ratio of $\kappa^{2} Q^{2}=0.18$. Use of this value in the $1 /\left(1+\kappa^{2} Q^{2}\right)$ reduction factor gives a predicted value of $30 \mathrm{~dB}$ growth for the

TABLE IV. Values of equivalent circuit components.

\begin{tabular}{lcc}
\hline \multicolumn{1}{c}{ Component } & Symbol & Value \\
\hline Capacitance of cavities & $C_{m}, C_{d}$ & $29.6 \mathrm{fF}$ \\
Inductance of cavities & $L_{m}, L_{d}$ & $137 \mathrm{nH}$ \\
Resistance of cavities & $R_{m}, R_{d}$ & $10 \Omega$ \\
Inductance of coupling loop & $L_{l}$ & $6.4 \mathrm{nH}$ \\
Resistance of coupling loop & $R_{l}$ & $0.01 \Omega$ \\
Mutual inductance between & $M$ & $2.7 \mathrm{nH}$ \\
loop and cavity & & \\
\hline \hline
\end{tabular}


coupled cavity experiments. This results in better agreement between theory and experiment than that reported in Ref. 4.

It might be argued that cavity coupling is equivalent to the often used BBU reduction technique of $Q$ lowering, because both methods serve to lower the strength of the $\mathrm{TM}_{110}$ mode fields. There is a significant difference, however. A finite $Q$ represents lossy processes from which energy cannot be recovered, whereas a nonzero $\kappa$ represents only reactive loading which does not result in any energy loss. If $Q$ were set to infinity, i.e., no dissipative losses, the process of power sharing would still exist and BBU growth rate reduction would still occur. ${ }^{17}$ Therefore, this technique provides a novel way in which to reduce the adverse effects of the BBU instability.

\section{CONCLUSIONS}

This paper contains the results of an experimental program whose only goal is to investigate the behavior of the BBU instability. Through the course of this research several original developments have been made regarding the physics of the BBU instability. The use of the continuum model was shown to accurately predict the growth of the BBU in a few (10) cavity system. The use of a more realistic discrete cavity model gave slightly better agreement. The importance of beam loading at high e-beam currents was demonstrated. Experimental BBU growth at increased current was found to be less than in theories (which do not include beam loading). The beam loading effect was mitigated when a cavity array employing lower $Q$ 's and lower frequency spread was used. Increasing the e-beam propagation distance had the expected effect on the BBU growth rate (increasing the slope of the growth vs current curve). The use of external cavity coupling as a novel BBU reduction technique was demonstrated, resulting in a $0.75 \mathrm{~dB}$ reduction per cavity. A model implementing power sharing through mutual inductance is capable of explaining the reduction with excellent agreement.

\section{ACKNOWLEDGMENTS}

This research was supported by Strategic Defense Initiative Office of Innovative Science and Technology man- aged by the Office of Naval Research. Support for P.R.M. was also supplied by a Rackham School of Graduate Studies Fellowship. Experimental assistance from M. T. Walter and C. H. Ching is acknowledged and appreciated.

${ }^{1}$ W. K. H. Panofsky and M. Bander, Rev. Sci. Instrum. 39, 206 (1968); A. W. Chao, B. Richter, and C. Y. Yao, Nuc. Instrum. Methods 178, 1 (1980).

${ }^{2}$ R. Helm and G. Loew, in Linear Accelerators, edited by P. M. Lapostelle and A. L. Septier (North-Holland, Amsterdam, 1970), Chap. B: 1.4, p. 173; A. W. Chao, Physics of Collective Beam Instabilities in High Energy Accelerators (Wiley, New York, 1993), p. 136.

${ }^{3}$ P. R. Menge, R. M. Gilgenbach, and R. A. Bosch, Appl. Phys. Lett. 61, 642 (1992).

${ }^{4}$ P. R. Menge, R. M. Gilgenbach, and Y. Y. Lau, Phys. Rev. Lett. 69, 2372 (1992).

${ }^{5}$ V. K. Neil, L. S. Hall, and R. K. Cooper, Part. Accel. 9, 213 (1979).

${ }^{6}$ G. J. Caporaso, F. Rainer, W. E. Martin, D. S. Prono, and A. G. Cole, Phys. Rev. Lett. 57, 1591 (1986).

${ }^{7}$ Y. Y. Lau, Phys. Rev. Lett. 63, (11), 1141, 2433 E (1989).

${ }^{8}$ R. M. Gilgenbach, L. D. Horton, R. F. Lucey, Jr., S. Bidwell, M. Cuneo, J. Miller, and L. Smutek, in Digest of the 5th IEEE Pulsed Power Conference (IEEE, New York, 1985), p. 126.

${ }^{9}$ V. K. Neil and R. K. Cooper, Part. Accel. 1, 111 (1970).

${ }^{10}$ D. G. Colombant and Y. Y. Lau, Appl. Phys. Lett. 55, 27 (1989).

${ }^{11}$ R. A. Bosch, P. R. Menge, and R. M. Gilgenbach, J. Appl. Phys.71, 3091 (1992).

${ }^{12}$ R. E. Shefer and G. Bekefi, Int. J. Electron. 51, 569 (1981).

${ }^{13}$ R. L. Gluckstern, F. Neri, and R. K. Cooper, Part. Accel. 23, 37 (1988).

${ }^{14}$ D. Chernin and A. Mondelli, Part. Accel. 24, 177 (1989).

${ }^{15}$ K. A. Thompson and R. D. Ruth, Phys. Rev. D 41, 964 (1990); R. H. Miller et al., SLAC Pub. 5862, 1992.

${ }^{16}$ C. L. Bohn and J. R. Delayen, Phys. Rev. A 25, 5964 (1992).

${ }^{17}$ D. Colombant, Y. Y. Lau, and D. Chernin, Part. Accel. 35, 193 (1991).

${ }^{18}$ D. G. Colombant and Y. Y. Lau, J. Appl. Phys. 72, 3874 (1992).

${ }^{19}$ D. G. Colombant and Y. Y. Lau, Nuc. Instrum. Methods A 311, 1 (1992).

${ }^{20}$ R. E. Collin, Foundations for Microwave Engineering (McGraw-Hill, New York, 1966), Chaps. 7 and 9.

${ }^{21}$ R. A. Bosch, P. R. Menge, and R. M. Gilgenbach (unpublished).

${ }^{22}$ P. R. Menge, Ph.D. dissertation, The University of Michigan, 1993.

${ }^{23}$ P. W. Tuinenga, SPICE, A Guide to Circuit Simulation and Analysis Using PSpice (Prentice-Hall, Englewood Cliffs, NJ, 1988). 